\title{
Importance of Defective and Non-Symmetric Structures in Silver Nanoparticles
}

David Loffreda*, Dawn M. Foster, Richard E. Palmer*, and Nathalie Tarrat*

David Loffreda - Univ Lyon, Ens de Lyon, CNRS UMR 5182, Université Claude Bernard Lyon 1, Laboratoire de Chimie, F-69342 Lyon, France

Dawn M. Foster - Nanoscale Physics Research Laboratory, School of Physics and Astronomy, University of Birmingham, Birmingham B15 2TT, UK

Richard E. Palmer - College of Engineering, Swansea University, Bay Campus, Fabian Way, Swansea SA1 8EN, U.K

Nathalie Tarrat - CEMES, Université de Toulouse, CNRS, 29, Rue Jeanne Marvig, 31055

Toulouse, France

AUTHOR INFORMATION

Corresponding Author

David Loffreda - Univ Lyon, Ens de Lyon, CNRS UMR 5182, Université Claude Bernard Lyon 1, Laboratoire de Chimie, F-69342 Lyon, France ; orcid.org/ 0000-0001-9912-7965; Email: david.loffreda@ens-lyon.fr

Richard E. Palmer - College of Engineering, Swansea University, Bay Campus, Fabian Way, Swansea SA1 8EN, U.K; orcid.org/ 0000-0001-8728-8083; Email: r.e.palmer@swansea.ac.uk 
Nathalie Tarrat - CEMES, Université de Toulouse, CNRS, 29, Rue Jeanne Marvig, 31055 Toulouse, France ; orcid.org/ 0000-0002-0909-0001; Email: nathalie.tarrat@ cemes.fr

ABSTRACT. Scanning Transmission Electron Microscopy experiments indicate that facecentered cubic (FCC) is the predominant ordered structure for Ag309 \pm 7 nanoclusters, synthesized in vacuum. Historically, experiments do not present a consensus on the morphology at these sizes, whereas theoretical studies find the icosahedral symmetry for Ag309 and the decahedral shape for nearby sizes. We employ density functional theory calculations to rationalize these observations, considering both regular and defective Ag nanoparticles (281-321 atoms). The change of stability induced by the presence of defects, symmetry loss and change of number of atoms is evaluated by the nanoparticle surface energy, which was measured previously. FCC and decahedral symmetries are found to be more favourable than icosahedral, consistent with our measurements of clusters protected from extended atmospheric exposure. Further, an energy-free descriptor, surface atomic density, is proposed and qualitatively reproduces the surface energy data. Non-symmetric and defective structures may be preferred over perfectly regular ones within a given size range.

\section{TOC GRAPHICS}

\section{Ag309 \pm 7 atoms ?}

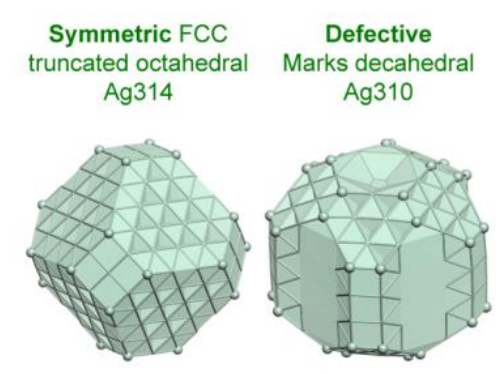

KEYWORDS. Silver Nanoparticles, Size-Selected Clusters, Defects, STEM, DFT, Surface Energy. 
The evolution of the crystalline structure of nanoparticles, especially noble and coinage transition metal clusters, as a function of the working conditions, chemical environment and size, is of great interest for applications in various domains of chemistry and physics. In this context the determination of the structural properties of silver nanoparticles has benefited from intensive research. However, the high oxidation and sulphidation propensity of silver makes the determination of the lowest energy isomer of pure clusters very challenging. ${ }^{1,2,3,4,5}$ At the experimental level, the conclusions regarding the preference of a specific symmetry (face-centered cubic FCC, icosahedral Ih and decahedral Dh) seems to depend essentially on the size of the nanoparticle. In fact, for large sizes $(45-140 \mathrm{~nm})$, all the highly symmetric structures have been synthesized in vacuum, showing no clear trend regarding relative stability. ${ }^{6}$ At lower sizes (1-5 $\mathrm{nm}$ ), a previous study devoted to size-selected Ag nanoparticles, synthesized by using the inert gas condensation technique, showed one preferential symmetry (Ih) over the others. ${ }^{7}$ At intermediate sizes (up to $11 \mathrm{~nm}$ ), both Ih and FCC symmetries have been observed explicitly. ${ }^{8}$ The authors concluded that the predominantly observed icosahedral symmetry at large size may be due to a kinetic trapping during the synthesis (the transformation from icosahedral to FCC symmetries costing too much energy). They suggested that some clusters below $2.5 \mathrm{~nm}$ may inhabit FCC symmetry due to a lower structural change activation barrier. According to these works, the most stable structure of Ag clusters related to the magic number 309 atoms is yet to be determined thoroughly. The theoretical studies aiming to tackle this question of the preference between all the high symmetries have been mostly developed by combining global optimization methods with semi-empirical potentials and examining clusters in the range 1-10000 atoms. ${ }^{9,10,11,12,13} \mathrm{~A}$ systematic study of $\mathrm{Ag}, \mathrm{Au}, \mathrm{Cu}$ and $\mathrm{Pd}$ nanoparticles of diameter up to $7 \mathrm{~nm}$ has been reported on the basis of Monte Carlo simulations using the EAM potential. ${ }^{12}$ According to their findings, the 
decahedral symmetry is predominant for Ag in the complete explored range of sizes, with a few exceptions for clusters exhibiting magic numbers, such as 309 , for which the icosahedral shape competes. In addition, the truncated octahedral symmetry is by far the least stable one. These results have confirmed previous similar studies reported by Baletto et al., especially for 309 atoms ${ }^{9,10}$ and by Doye and Calvo, ${ }^{11}$ showing the preference for decahedral symmetry in the range 240-36600 atoms at $0 \mathrm{~K}$. These works thus agree on the preference of decahedral forms in the range 1-10000 atoms, with the exception of magic numbers, such as 309 , where the icosahedral cluster is favored. However, one study based on the GUPTA semi-empirical potential and modified dynamic lattice search showed that the Marks decahedral structure is more favorable than the icosahedron for 309 atoms. ${ }^{13}$ In summary, the experimental results do not show a clear consensus for the structure of Ag nanoparticles in a large range of sizes (1-140 nm), whereas theoretical findings, exclusively based on semiempirical potentials and global optimization methods, consistently conclude a preference for decahedral shapes below 10000 atoms, with the exception of magic numbers. Hence the discrepancies between theory and experiment require at the experimental level a thorough investigation of the stability of Ag clusters, in particular for 309 atoms, for which there is a lack of statistics combining all the competitive symmetries and the presence of defects. Moreover, accurate theoretical calculations within a quantum mechanical formalism, such as Density Functional Theory (DFT), are clearly missing for metallic nanoparticles of several nanometers. To advance on these questions, we have combined in an original way synthesis and characterization of size-controlled Ag nanoparticles (309 \pm 7 atoms), including cluster symmetry distribution and statistics, with DFT calculations on comparable cluster sizes (281-321 atoms) considering both symmetric, non-symmetric and defective morphologies. 
Regarding synthesis, samples of size-selected Ag clusters were produced using a magnetron sputtering, gas aggregation cluster beam source ${ }^{14}$ with lateral time-of-flight mass filter, ${ }^{15}$ by following our previous studies for Au clusters ${ }^{16}$ (see section 1.1 of the Supporting Information for the methods). The atomic structures of the clusters were again characterised using sphericalaberration corrected Scanning Transmission Electron Microscopy in High Angle Annular Dark Field (STEM-HAADF) mode. The isomer symmetries observed in the experimental images were identified by using the Simulation Atlas method. ${ }^{17,18,19,20}$ Significantly, due to the known sensitivity of silver to air, samples were transferred between the vacuum load-lock of the cluster deposition source and the STEM in an inert gas glove-box. This reduces the duration of ambient exposure to about 5 seconds. The distribution of atomic structures for size-selected Ag clusters containing $309 \pm 7$ atoms is presented (Figure 1a) together with example images obtained by STEM-HAADF (Figure 1b-e). Surprisingly, FCC is the dominant symmetry (40\%), followed by Dh (13\%), with very few clusters exhibiting the Ih symmetry (2\%) (Figure 1a). The relatively proportion of unidentified/amorphous (UI/A) clusters at this size (45\%) results from the increased fluctuations (rotations and structural changes) of such small clusters from frame to frame, ${ }^{21,22}$ amplified by electron beam heating, during the acquisition of a single frame, which takes about a second. The set of UI/A clusters includes all the non-crystalline, quasi-crystalline and purely amorphous nanoparticles whose structure cannot be characterized. The two main ordered structures (FCC and Dh) are illustrated in Figure 1b,c for FCC and Figure 1d,e for Dh. Although the core structures are clear, the images of those two nanoparticles (Figure 1c,e) show some defects. The clusters present several irregularities: local symmetry loss, missing atoms or parts and extended imperfections. We conclude that FCC predominates at Ag309 \pm 7 , whereas the conventional experimental wisdom is $\mathrm{Ih}$ symmetry, ${ }^{7}$ the latter consistent with a previous 
theoretical study based on a semiempirical potential reporting on the preference of Ih symmetry at 309 atoms, or Dh for a wide range of sizes. ${ }^{12}$
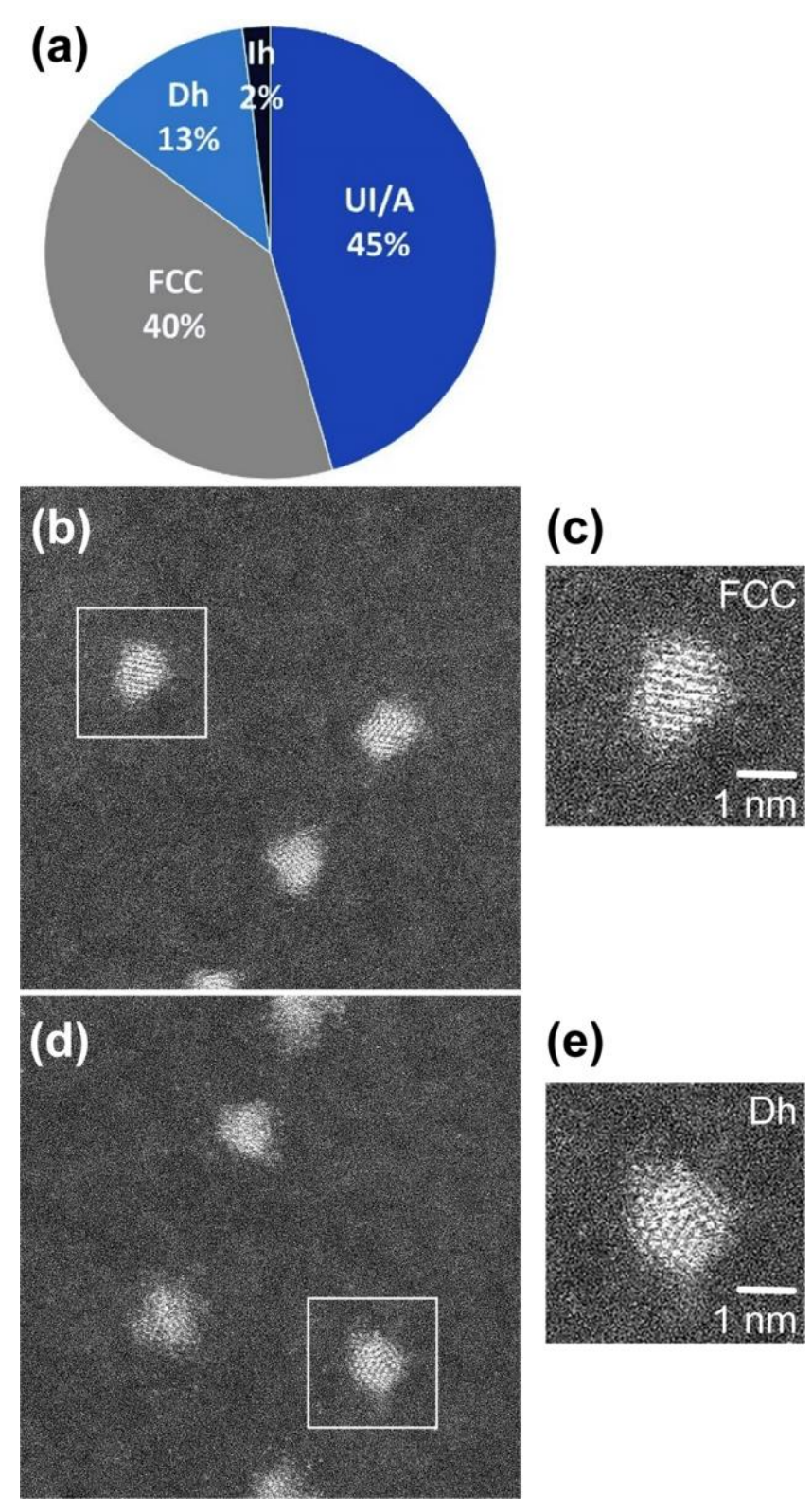

(e)

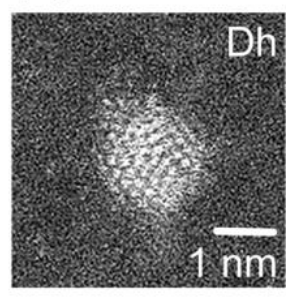

Figure 1. (a) Distribution statistics of Ag cluster symmetries around 309 atoms. The structures are identified as FCC, Dh, Ih and unidentified or amorphous (UI/A). (b)-(e) STEM-HAADF images at $293 \mathrm{~K}$ illustrating (b) and (d) a series of nanoparticles of various symmetries. (c) FCC and (e) Dh structures were extracted from (b) and (d), respectively (see white squares). 
In order to investigate further this discrepancy between our measurements and the literature, we propose a rational based on theoretical models inspired by all the available experimental data (size, symmetry and presence of defects). Our goal is to evaluate the relative stability of a limited family of clusters of which the number of atoms changes, thus probing the importance of defects and notsymmetrically grown structures, rather than determining the global minimum at a given size. In this framework, we have performed DFT calculations (see the section 1.2 of the Supporting Information for computational details) of sixteen silver clusters of size around 309 atoms, exhibiting either regular FCC, Dh and Ih structures or structural variations around high symmetries (defective and non-symmetrically grown nanoclusters). The cluster stability is determined at the DFT-D3 level, by calculating the nanoparticle surface energy, which can be validated with respect to available measurements (see the section 1.3 of the Supporting Information for a justification of the choice of the dispersion-corrected exchange-correlation DFT functional and for the formula of the nanoparticle surface energy). In our study, this surface energy is determined by calculating the nanoparticle excess energy normalized to the surface area (see Eq. (S3) of the Supporting Information). For silver nanoparticles, the surface energy has been measured by Blackman et al. $\left(1.13 \mathrm{~J} . \mathrm{m}^{-2}\right),{ }^{23}$ based on the Kelvin equation model. Among the available experimental data, ${ }^{24}$ this surface energy is compatible with measured values of $\mathrm{Ag}$ crystalline surfaces, ${ }^{25}$ but also with recent measurements for $\mathrm{Au}, \mathrm{Cu}$ and $\mathrm{AuCu}$ nanoparticles. ${ }^{26}$

The sixteen studied isomers (Figure 2 and section 2 of the Supporting Information), which contain between 281 and 321 silver atoms, can be divided into 5 structural families and four symmetries including icosahedra (Ih, noted ico), ino-decahedra (D5h, noted ino), Marks decahedra (D5h, noted marks), cuboctahedra (Oh, noted cubo), and truncated octahedra (Oh noted ito if irregular hexagonal facets are present). Note in passing that although truncated octahedron and 
cuboctahedron present different atomic structures, their octahedral symmetry Oh comes from the FCC packing, thus meaning that such a distinction cannot be done in our measurements. Defective crystalline and non-crystalline nanoclusters (truncated cuboctahedra noted tcubo, defective truncated octahedra noted dto and defective Marks decahedra noted dmarks) have also been modeled either by keeping the related initial symmetry of the non-defective cluster (Dh or Oh) or by losing the symmetry completely $\left(\mathrm{C}_{1}\right)$. These defective clusters are generally expected to be less stable than highly symmetrical forms, thus explaining why they are never considered. This set of nanoclusters (Figure 2) offers also a wide range of possibilities for the facets: (111), (100), (110)$(1 \times 1),(110)-(1 \times 2),(211),(311)$ and $(331)$. 

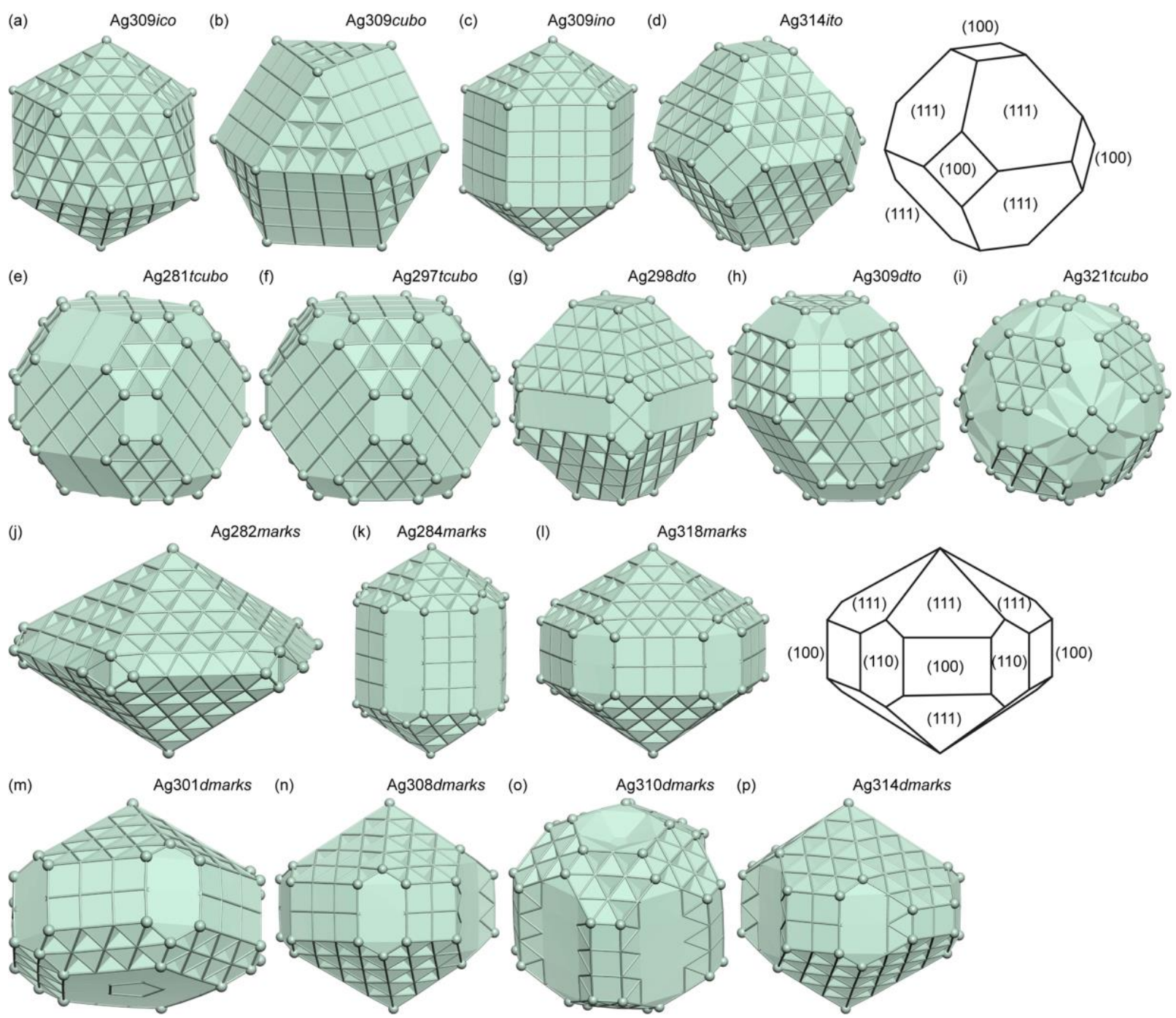

Figure 2. 3D views of sixteen Ag nanoclusters optimized at the DFT-D3 level. The vertices of the nanoclusters are depicted with balls while atoms belonging to edges and facets are drawn with sticks. Perfectly regular and convex Ag309 (a) icosahedron, (b) cuboctahedron and (c) inodecahedron. (d) Convex Ag314 truncated octahedron with irregular (111) hexagonal and regular (100) square facets. Truncated cuboctahedra containing (e) 281, (f) 297 (coming from Ag309cubo, both presenting (100) and (111) convex facets and (110)-(1x1) concavities) and (i) 321 atoms (with convex (100), (111) and concave (110)-(1×1), (311) facets). Defective truncated octahedra with (g) 298 (coming from Ag314ito and with (100), (111) and (110)-(1×1) facets) and (h) 309 atoms 
(with convex (100), (111) and concave (110)-(1×1), (211) facets). Regular Marks decahedra (j) Ag282 (with convex (111) and concave (110)-(1×1) facets), (k) Ag284 and (1) Ag318 (with convex (100), (111) and concave (110)-(1×1) facets). Defective Marks decahedra with (m) 301, (n) 308 (both coming from Ag318marks), (o) 310 and (p) 314 atoms. (m) Ag301dmarks: a decahedral ending part has been cut over several layers, resulting in a pentagonal facet composed of pentagonal concentric trough and cavity. (n) Ag308dmarks: a fifth of the lateral (110)-(1×1) concavities has been extended to a deeper trough mimicking a surface $(110)-(1 \times 2)$ missing-row reconstruction. (o) Ag310dmarks: all the lateral concavities have been symmetrized following a missing-row reconstructed $(110)-(1 \times 2)$ pattern and the two decahedral ending parts have been cut to form pentagonal cavities. (p) Ag314dmarks: the lateral (110) concavities are of different nature, (110)-(1×1), missing-row reconstructed (110)- $(1 \times 2)$ and stepped $(331)$.

The nanoparticle surface energy is defined in this work as the excess energy normalized to the nanoparticle surface area (a larger stability being related to a minimal surface energy). This area based on the computed structures has been approximated by considering symmetrized and planar facets and using the average metal-metal distance at the surface of nanoparticles (see section 3.1 of the Supporting Information for more details and section 3.2 for the analytical formulas). For evaluating these areas, four methods have been tested and compared with measurements as detailed in the sections 3.3.1 and 3.3.2 of the Supporting Information (methods $\mathrm{n}^{\circ} 1-3$ have been introduced previously ${ }^{27}$ and method $n^{\circ} 4$, which is introduced here, corresponds to a refinement of method $\mathrm{n}^{\circ} 2$ ). According to this analysis in the range 1-3.5 nm for Ag clusters, the method $\mathrm{n}^{\circ} 4$ has been retained for our study since it is the most predictive one. Based on the areas computed with the latter method, the DFT surface energies of the sixteen nanoclusters (Figure 3) vary in the range 
0.85-1.20 J.m $\mathrm{m}^{-2}$, inside an interval of $0.33 \mathrm{~J} . \mathrm{m}^{-2}$ with an average value of $1.04 \mathrm{~J} . \mathrm{m}^{-2}$. The latter value calculated in the range $2-2.5 \mathrm{~nm}$ is in fair agreement with Blackman et al.'s measurements (1.13 $\left.\mathrm{J}_{\mathrm{m}} \mathrm{m}^{-2}\right)^{23}$. For large nanoparticles, the approximation of infinite facets holds. The most likely surface terminations have been modeled by symmetric slabs composed of 19 layers for $\mathrm{Ag}(111)$ and (100) and 27 layers for $\mathrm{Ag}(110)$ at PBE-D3 level (see section 1.3 of the Supporting Information). The corresponding surface energies are $1.25,1.31$ and $1.39 \mathrm{~J} . \mathrm{m}^{-2}$, respectively, in fair agreement with experiments operated on Ag polycrystalline surfaces $\left(1.32 \mathrm{~J} . \mathrm{m}^{-2}\right)^{25}$. According to measurements, the nanoparticle surface energy increases with the size of the nanoparticle from 1.13 to $1.32 \mathrm{~J}^{-2} \mathrm{~m}^{-2}$. This trend is quantitatively reproduced by our computed surface energies.

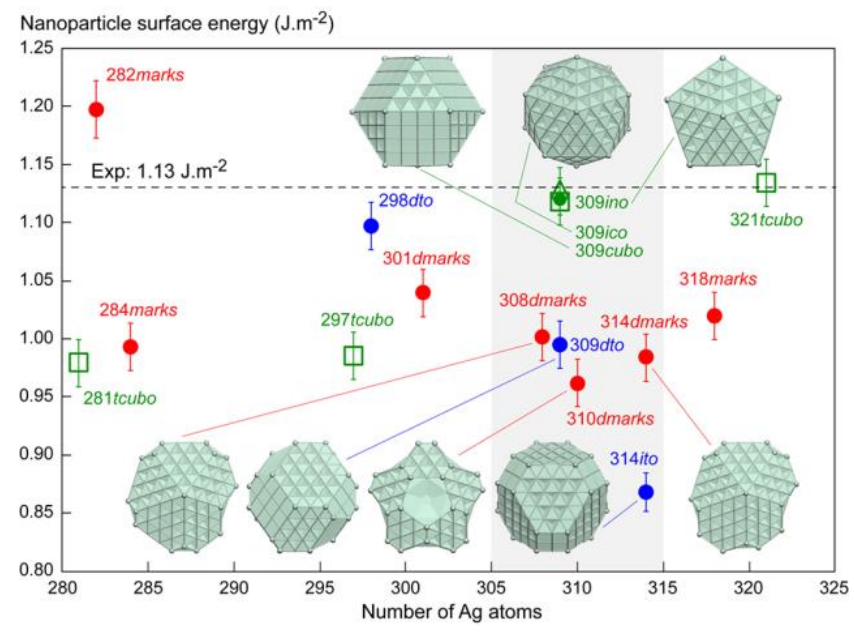

Figure 3. DFT-D3 nanoparticle surface energies $\left(\mathrm{J} . \mathrm{m}^{-2}\right)$ as a function of the number of Ag atoms for the set of sixteen nanoparticles (Figure 2). Standard deviations due to systematic error of the chosen method on the structure are indicated by vertical intervals (see section 3.3 .3 of the supporting information for their evaluation). The vertices of the nanoclusters are depicted with balls while atoms belonging to edges and facets are drawn with sticks. The experimental value $1.13 \mathrm{~J} . \mathrm{m}^{-2}$ (dotted line) is extracted from the literature. ${ }^{23}$ 
Three types of morphologies are competitive in the explored range 281-321 atoms: truncated cuboctahedra, irregular and defective truncated octahedra and regular and defective Marks decahedra. There are three exceptions: Ag282marks, Ag298dto and Ag321tcubo corresponding to defective nanoclusters with high instabilities (presenting either atomic-sized cavities or a significant proportion of concave $(110)-(1 \times 1)$ or kinked \& stepped facets). The regular trio Ag309cubo, Ag309ico and Ag309ino present a similar stability in surface energy following the order $c u b o>$ ico > ino, while the absolute stability in total electronic energy gives the following trend ico $>$ ino $=$ cubo. Interestingly, the surface energies of these latter three nanoparticles are larger than those of the three competitive morphologies. Hence the icosahedral cluster is less favorable than truncated octahedral and Marks decahedral structures on the basis of this energetic analysis. In the experimental range $309 \pm 7$ atoms, 8 nanoclusters have been optimized. Among them, Ag314ito is the preferential one. Then four defective truncated octahedron (Ag309dto) and Marks decahedra (Ag308dmarks, Ag310dmarks, Ag314dmarks) compete in a narrow range of surface energy. Hence, at least five Dh and FCC structures are more favorable in surface energy than the icosahedron in the range $309 \pm 7$ atoms, in fair agreement with our measurements (Figure 1), showing the predominance of FCC clusters over Dh isomers. Note that the cluster presenting the lowest surface energy (Ag314ito) is a FCC isomer in our theoretical study. These predicted trends of relative stability between nanoclusters are not dependent on the deviations due to systematic errors as illustrated in Figure 3 and demonstrated in the section 3.3.3 of the Supporting Information.

Such a preference for FCC symmetry can now be compared with the literature. From the experimental standpoint, the major differences with previous measurements concern first, the weak presence of icosahedral structures in our study and second, the observation of decahedral shapes 
which have never been reported previously. As a matter of fact, the two key earlier studies have concluded rather on the existence of icosahedral clusters especially in the range 1-5 $\mathrm{nm} .^{7,8} \mathrm{In}$ both these previous studies, it was suggested that the presence of large Ih clusters was a result of kinetic trapping and template growth on smaller seed structures. Reinhard et al. ${ }^{8}$ suggested that the growth conditions in the gas aggregation source are responsible for the kinetic trapping; specifically, the formation of large Ih clusters was attributed to rapid growth as the temperature dropped far from the evaporation source. In the present work, we employ growth conditions in the gas aggregation source that are known to reduce kinetic trapping, ${ }^{16}$ namely long condensation length and low magnetron power. These formation conditions allow for longer growth time, and hence more equilibrated structures. Our experimental approach and STEM-HAADF measurements lead us to conclude that the most frequently observed isomer in the population (FCC) is the ground state structure at this size, hence supporting Reinhard et al.'s previous work, where the FCC structure had been evoked with no clear demonstration. ${ }^{8}$ More interestingly, the existence of favored FCC forms at a size of $2.2 \mathrm{~nm}$ is also original since the previous literature was evidencing it for large sizes. ${ }^{8}$ Besides, according to our statistics, the preference of decahedral symmetry over the icosahedral one is original with respect to the experimental state of the art.

From the theoretical standpoint, our experimental conclusions do not support previous results arising from global optimization method and concluding to the predominance of decahedral symmetry on a wide range of sizes, with a competition with icosahedral forms for magic sizes (in particular 309 atoms). ${ }^{9,10,11,12,13}$ At least, our measurements confirm the existence of decahedral symmetry. In contrast, the biggest difference between our experimental statistics at $2.2 \mathrm{~nm}$ and previous theoretical works, is the predominance of FCC symmetry, which has been found only once and in a minority fraction by Rahm and Erhart. ${ }^{12}$ 
A further analysis based on our theoretical study confirms the preference of FCC and decahedral clusters over icosahedral form, which is in partial agreement with global optimization studies showing the predominance of decahedral structures in a large range of sizes. It is difficult to extend this particular discussion since our static and Heuristic theoretical approach is based on density functional theory in accurate computational conditions, whereas most of the systematic and previous studies (Monte Carlo simulations) use semi-empirical effective potentials. Interestingly, our theoretical models clearly show the importance of considering defective structures and nonsymmetrically grown nanoparticles, which are rarely discussed in the theoretical literature.

The discussed theoretical results are based on accurate DFT calculations and surface energies so far. To propose an alternative descriptor allowing us to discuss qualitatively the relative stability of the $\mathrm{Ag}$ nanoclusters, we introduce the surface atomic density (Figure 4). This is calculated by dividing the number of surface atoms of the outer shell of the nanoparticles by the nanoparticle surface area (calculated as previously). This energy-free descriptor, easily computed for each structure, can be considered as an approximation of the variation of the coordination of surface atoms, ${ }^{28}$ with respect to the nanoparticle core (normalized to surface area). In fact, the number of metallic bonds per atom varies between the core of the nanoparticle and the surface, in quantity (due to the formation of the surface) and in quality (due to the nanocluster morphology). This change of number of metallic bonds in the surface is defined as a coordination loss which is linked to a loss of total electronic energy. Hence the change of the number of surface atoms (named here surface atomic density) is indirectly correlated to the cluster stability. 


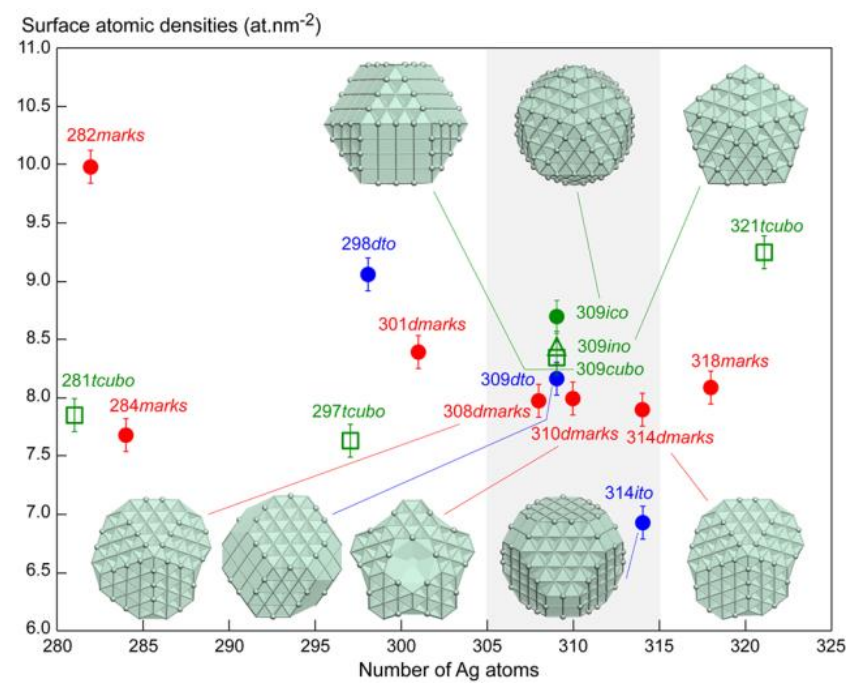

Figure 4. Surface atomic densities $\left(\right.$ at. $\mathrm{nm}^{-2}$ ) as a function of the number of Ag atoms. Standard deviations due to systematic error of the chosen method on the surface areas are indicated by vertical intervals (see section 3.3.3 of the supporting information). The atoms belonging to edges of the nanoclusters are depicted with balls while those belonging to vertices and facets are drawn with sticks.

Equivalent trends are observed when comparing the predicted nanoparticle surface energies (Figure 3) with the surface atomic densities (Figure 4). We show that there exists a correlation between these two descriptors. In fact, the relative order between all the nanoclusters is qualitatively reproduced with some exceptions around 309 atoms: the trio Ag309cubo, ico, ino for which the surface atomic density suggests weaker differences with respect to Ag314ito. The limits of surface atomic densities can also be seen in the relative positions of the trio Ag309cubo, ico, ino by comparison with surface energies (the number of surface atoms being identical for these three nanoclusters). From a general standpoint, small deviations are observed between the theoretical trends, but not changing the overall order, with Marks decahedral and truncated octahedral structures having lower surface atomic densities than the icosahedron. 
In this work, a combined experimental and theoretical analysis of synthesized Ag nanoparticles imaged by STEM-HAADF and modeled by DFT has been proposed to investigate the competition between several symmetries and nanoparticle morphologies around the given size 309 atoms. The experimental statistics suggests a predominance of FCC clusters followed by decahedral symmetry and very minority icosahedral forms. The relative stability of a finite set of Ag nanoclusters including symmetric, non-symmetric and defective clusters in the range 281-321 atoms has been determined by surface energy calculations, at the dispersion-corrected PBE-D3 level. The lowest surface energy structures are those of FCC and decahedral symmetries, in fair agreement with our measurements. The icosahedral shape has never been found competitive neither from experiments nor from theory. Our quantitative theoretical analysis has been extended by a qualitative model based on surface atomic density, an energy-free descriptor which reproduces the surface energy trends. In conclusion, this work shows the preference of FCC and decahedral symmetries for Ag clusters close to 309 atoms. In addition, the non-symmetrically grown or defective clusters may be more stable than perfectly symmetric ones, in agreement with our experimental observations. These original and unexpected structures have to be calculated systematically and compared to usual and symmetrical clusters, when the goal is the determination of the global minimum at a given size. This theoretical work based on a static approach and compared to measurements opens interesting perspectives for investigating structural transitions between competitive forms and temperature effects for a finite set of $\mathrm{Ag}$ nanoclusters, by molecular dynamics and approximated DFT methods. 


\section{ASSOCIATED CONTENT}

\section{Supporting Information}

Experimental section, methods and computational details are exposed with the definition of nanoparticle surface energy and the methods for calculating the nanoparticle surface areas. Complementary theoretical results, optimized structures and analyses are reported.

(1) Table S1 justification of the chosen DFT functional

(2) Figure S1-S16 3D views of optimized DFT structures of 16 Ag nanoparticles

(3) Table S2 analytical formulas of nanoparticle surface areas

(4) Table $\mathbf{S 3}$ experimental reference for nanoparticle surface areas

(5) Figure S17 comparative analysis of several methods for evaluating the surface areas

(6) Figure S18 expected deviations of surface energies and surface areas for the Ag nanoclusters

(7) Cartesian coordinates of optimized DFT structures of 16 Ag nanoparticles

\section{AUTHOR INFORMATION}

\section{Notes}

The authors declare no competing financial interests.

\section{ACKNOWLEDGMENTS}

The authors thank IDRIS in Paris, CINES in Montpellier, TGCC in Grenoble (project 609, GENCI/CT8) and PSMN in Lyon for CPU time and assistance. The authors thank the CPER/SYSPROD 2015-2022 project (N²019-AURA-P5B), AXELERA Pôle de Compétitivité (PSMN Data Center), EPSRC and NERC for financial supports. This work was granted access to 
the HPC resources of CALMIP supercomputing center under the allocations 2019-[p19067] and 2020-[p1303].

\section{REFERENCES}

(1) Reagor, B. T.; Sinclair, J. D. Tarnishing of Silver by Sulfur Vapor: Film Characteristics and Humidity Effects. J. Electrochem. Soc. 1981, 128, 701.

(2) Bennett, J. M.; Stanford, J.; Ashley, E. Optical Constants of Silver Sulfide Tarnish Films. J. Opt. Soc. Am. 1970, 60, 224-232.

(3) Yin, Y.; Li, Z-Y.; Zhong, Z.; Gates, B.; Xia Y.; Venkateswaranc, S. Synthesis and characterization of stable aqueous dispersions of silver nanoparticles through the Tollens process. J. Mater. Chem. 2002, 12, 522-527.

(4) Erol, M.; Han, Y.; Stanley, S. K.; Stafford, C. M.; Du, H.; Sukhishvili, S. SERS not to be taken for granted in the presence of oxygen. J. Am. Chem. Soc. 2009, 131, 7480-7481.

(5) Matikainen, A.; Nuutinen, T.; Itkonen, T.; Heinilehto, S.; Puustinen, J.; Hiltunen, J.; Lappalainen, J.; Karioja P.; Vahimaa, P. Atmospheric oxidation and carbon contamination of silver and its effect on surface-enhanced Raman spectroscopy (SERS). Sci. Rep. 2016, 6, 37192.

(6) Barke, I.; Hartmann, H.; Rupp, D.; Flückiger, L.; Sauppe, M.; Adolph, M.; Schorb, S.; Bostedt, C.; Treusch, R.; Peltz, C. et al., T. The 3D-architecture of individual free silver nanoparticles captured by X-ray scattering. Nature Communications 2015, 6, 6187.

(7) Gracia-Pinilla, M.; Ferrer D.; Mejía-Rosales S.; Pérez-Tijerina, E. Size-selected Ag nanoparticles with five-fold symmetry. Nanoscale Res Lett. 2009, 4, 896-902. 
(8) Reinhard, D.; Hall, B. D.; Ugarte, D.; Monot, R. Size-independent fcc-to-icosahedral structural transition in unsupported silver clusters: An electron diffraction study of clusters produced by inert-gas aggregation. Phys. Rev. B 1997, 55, 7868-7881.

(9) Baletto, F.; Mottet, C.; Ferrando, R. Microscopic mechanisms of the growth of metastable silver icosahedra. Phys. Rev. B 2001, 63, 155408.

(10) Baletto, F.; Ferrando, R.; Fortunelli, A.; Montalenti, F.; Mottet, C. Crossover among structural motifs in transition and noble-metal clusters. J. Chem. Phys. 2002, 116, 3856-3863.

(11) Doye, J. P. K.; Calvo, F. Entropic Effects on the Size Dependence of Cluster Structure. Phys. Rev. Lett. 2001, 86, 3570.

(12) Rahm, J. M.; Erhart, P. Beyond Magic Numbers: Atomic Scale Equilibrium Nanoparticle Shapes for Any Size. Nano Lett. 2017, 17, 5775-5781.

(13) Shao, X.; Yang, X.; Cai, W. Geometry optimization and structural distribution of silver clusters from $\mathrm{Ag}_{170}$ to $\mathrm{Ag}_{310 .}$ Chem. Phys. Lett. 2008, 460, 315-318.

(14) Pratontep, S.; Carroll, S. J.; Xirouchaki, C.; Streun, M.; Palmer, R. E. Size-selected cluster beam source based on radio frequency magnetron plasma sputtering and gas condensation. Rev. Sci. Instrum. 2005, 76, 045103.

(15) von Issendorff, B.; Palmer R. E. A new high transmission infinite range mass selector for cluster and nanoparticle beams. Rev. Sci. Instrum. 1999, 70, 4497-4501.

(16) Plant, S. R.; Cao, L.; Palmer, R. E. Atomic Structure Control of Size-Selected Gold Nanoclusters during Formation. J. Am. Chem. Soc. 2014, 136, 7559.

(17) Wang, Z. W.; Palmer R. E. Determination of the ground-state atomic structures of sizeselected Au nanoclusters by electron-beam-induced transformation. Phys. Rev. Lett. 2012, 108, 245502. 
(18) Wells, D. M.; Rossi, G.; Ferrando, R.; Palmer R. E. Metastability of the atomic structures of size-selected gold nanoparticles. Nanoscale 2015, 7, 6498-6503.

(19) Jian, N.; Palmer, R. E. Variation of the core atomic structure of thiolated $\left(\mathrm{Au}_{\mathrm{x}} \mathrm{Ag}_{1-\mathrm{x}}\right)_{(312 \pm 55)}$ nanoclusters with composition from aberration-corrected HAADF STEM. J. Phys. Chem. C 2015, $119,11114-11119$.

(20) Hu, K. J.; Plant, S. R.; Ellis, P. R.; Brown, C. M.; Bishop, P. T.; Palmer R. E. Atomic resolution observation of a size-dependent change in the ripening modes of mass-selected $\mathrm{Au}$ nanoclusters involved in CO oxidation. J. Am. Chem. Soc. 2015, 137, 15161-15168.

(21) Wang, Z. W.; Palmer, R. E. Direct atomic imaging and dynamical fluctuations of the tetrahedral Au20 cluster. Nanoscale 2012, 4, 4947.

(22) Wang, Z. W.; Palmer, R. E. Experimental evidence for fluctuating, chiral-type Au55 clusters by direct atomic imaging. Nano Lett. 2012, 12, 5510-5514.

(23) Blackman, M.; Lisgarten, N. D.; Skinner, L. M. Surface Energy and Evaporation Rate of Spherical Particles of Radii less than 500 A. Nature 1968, 217, 1245-1246.

(24) Nanda, K. K.; Maisels, A.; Kruis, F. E.; Fissan, H.; Stappert S. Higher Surface Energy of Free Nanoparticles. Phys. Rev. Lett. 2003, 91, 106102.

(25) Tyson, W. R.; Miller, W. A. Surface Free Energies Of Solid Metals Estimation From Liquid Surface Tension Measurements. Surf. Sci. 1977, 62, 267-276.

(26) Chmielewski, A.; Nelayah, J.; Amara, H.; Creuze, J. ; Alloyeau, D.; Wang, G.; Ricolleau, C. Direct Measurement of the Surface Energy of Bimetallic Nanoparticles: Evidence of Vegard's Rulelike Dependence. Phys. Rev. Lett. 2018, 120, 025901. 
(27) Molleman, B. ; Hiemstra, T. Size and shape dependency of the surface energy of metallic nanoparticles: unifying the atomic and thermodynamic approaches. Phys. Chem. Chem. Phys. 2018, 20, 20575.

(28) Jiang, Q.; Lu, H. M.; Zhao, M. Modelling of surface energies of elemental crystals. J. Phys.: Condens. Matter 2004, 16, 521. 